\title{
Concurrent administration of trastuzumab and anthracyclines as adjuvant regimen for HER2-positive breast cancer: a randomised controlled trial
}

\author{
Songjie Shen ${ }^{1, *}$, Ying $\mathrm{Xu}^{1,{ }^{1}}$, Yidong Zhou ${ }^{1}$, Feng Mao ${ }^{1}$, Jinghong Guan ${ }^{1}$ and Qiang \\ Sun ${ }^{1}$ \\ ${ }^{1}$ Department of Breast Surgery, Peking Union Medical College Hospital, Peking Union Medical College, Chinese Academy of \\ Medical Sciences, 1 Shuaifuyuan, Dongcheng District, Beijing 100730, China \\ *These authors have contributed equally to this work \\ Correspondence to: Qiang Sun, email: sunqiangpumc@sina.com \\ Keywords: breast cancer, adjuvant therapy, trastuzumab, anthracycline, cardiac safety \\ Received: June 22, $2017 \quad$ Accepted: August 28, $2017 \quad$ Published: October 06, 2017 \\ Copyright: Shen et al. This is an open-access article distributed under the terms of the Creative Commons Attribution License 3.0 \\ (CC BY 3.0), which permits unrestricted use, distribution, and reproduction in any medium, provided the original author and source \\ are credited.
}

\section{ABSTRACT}

Background: The regimen of concurrent administration of trastuzumab and anthracyclines in the adjuvant treatment of breast cancer has never been evaluated prospectively for fear of cardiac toxicity.

Methods: Patients with HER2-positive operable breast cancer were randomised to receive adjuvant treatment with concurrent or sequential administration of trastuzumab and anthracyclines. Cardiac monitoring was scheduled at baseline and every $\mathbf{3}$ months after the first dose of trastuzumab. The primary study endpoint was cardiac safety. Secondary endpoints were disease-free and overall survival.

Results: From 2011 to 2014, 201 participants were enrolled and randomised. The median follow-up time was 42 months. Nineteen patients (19.4\%) in the concurrent group and 22 patients (22.4\%) in the sequential group met the criteria for cardiac events with non-significant difference $(P=0.598)$. There was no difference in the mean LVEF between the two groups at the baseline and at 3, 6, 9, 12, and 24 months after the first dose of trastuzumab. No case of congestive heart failure or cardiac death occurred. The differences between the efficacies of the two regimens, defined by disease-free or overall survival, were not significant.

Conclusions: Concurrent administration of trastuzumab and anthracyclines is a safe adjuvant regimen and it provides evidence for further clinical trials.

\section{INTRODUCTION}

The human epidermal growth factor receptor 2 (HER2) is overexpressed in approximately $15 \%-25 \%$ of invasive breast cancers, and is associated with a high risk of disease recurrence and reduced survival [1-3]. Trastuzumab, a monoclonal antibody directed against HER2, has been shown to improve disease-free survival (DFS) and overall survival (OS) in HER2-positive breast cancer patients [1-3]. Moreover, many clinical trials have also indicated that patients with HER2-positive breast cancer might derive preferential benefit from anthracyclines [4-6].

However, cardiac dysfunction is the most concerning toxicity associated with these regimens, especially when trastuzumab is given concurrently with anthracyclines. In a pivotal trial in the metastatic setting, the concurrent administration of trastuzumab and anthracyclines was reported to result in unacceptably high rates of cardiac dysfunction (27\%), although the highest survival benefit was also observed in this treatment [7]. However, in the neoadjuvant therapy, trastuzumab administered concurrently with anthracyclines has shown exceedingly 
low cardiotoxicity [8-11], and expectedly high rates of pathological complete response $(\mathrm{pCR})$ and prolonged survival [8-10].

Although the findings of the North Central Cancer Treatment Group N9831 trial indicated an increase in disease-free survival with concurrent trastuzumab and paclitaxel compared to sequential regimen in the adjuvant treatment of breast cancer [12], only the sequential regiment of trastuzumab and anthracyclines administration was evaluated in prospective clinical trials. To date, the safety and efficacy of trastuzumab administered concurrently with anthracyclines has never been evaluated prospectively in the adjuvant treatment. Concurrent administration of trastuzumab and anthracyclines was not recommended in the adjuvant therapy of breast cancer in all the guidelines, including the latest version of the National Comprehensive Cancer Network (NCCN) guidelines of breast cancer (version 2.2016) and the American Society of Clinical Oncology (ASCO) guideline adaptation of the Cancer Care Ontario clinical practice guideline [13], because fear of cardiac toxicity. Thus, we conducted the present prospective, randomised, and controlled trial to assess the cardiac safety, as well as the therapeutic efficacy, of the concurrent administration of trastuzumab and anthracyclines in the adjuvant treatment of HER2-positive early breast cancer.

\section{RESULTS}

\section{Patient characteristics}

A total of 201 patients were enrolled (101 in the concurrent group and 100 in the sequential group) in the investigation from August 10, 2011 to March 1, 2014 (Figure 1). The last follow-up date was August 1, 2016, and the median follow-up time was 42 months (range: 19-62 months). Three of these patients in the concurrent group and two participants in the sequential group withdrew consent before starting treatment. Therefore, 196 participants (98 in the concurrent group and 98 in the sequential group) were eligible for final analysis. Table 1 showed the baseline characteristics for the concurrent and sequential groups. Characteristics were well balanced between the two treatment groups.

\section{1 patients with HER2-positive breast cancer randomized}

\begin{tabular}{|c|c|}
\hline $\begin{array}{l}101 \text { allocated to concurrent } \\
\text { administration of trastuzumab } \\
\text { and anthracyclines }\end{array}$ & $\begin{array}{l}100 \text { allocated to sequential } \\
\text { administration of trastuzumab } \\
\text { and anthracyclines }\end{array}$ \\
\hline $\begin{array}{l}98 \text { received allocated treatment } \\
3 \text { did not received allocated } \\
\text { treatment } \\
3 \text { withdrew consent }\end{array}$ & \begin{tabular}{|l}
98 received allocated treatment \\
2 did not received allocated \\
treatment \\
2 withdrew consent \\
\end{tabular} \\
\hline $\begin{array}{l}2 \text { temporarily withheld } \\
\text { trastuzumab } \\
1 \text { discontinued trastuzumab }\end{array}$ & $\begin{array}{l}1 \text { temporarily withheld } \\
\text { trastuzumab } \\
0 \text { discontinued trastuzumab }\end{array}$ \\
\hline
\end{tabular}

Figure 1: CONSORT diagram. HER2, human epidermal growth factor receptor. 
Table 1: Demographic and baseline characteristics

\begin{tabular}{|c|c|c|c|}
\hline Variable, N (\%) & $\begin{array}{c}\text { Concurrent group } \\
(\mathrm{n}=98)\end{array}$ & $\begin{array}{c}\text { Sequential group } \\
(\mathrm{n}=98)\end{array}$ & $P$-value \\
\hline \multicolumn{4}{|l|}{ Age(years) } \\
\hline Mean \pm SD & $48.0 \pm 9.0$ & $48.7 \pm 9.5$ & 0.628 \\
\hline Median (range) & $49.5(25 \sim 67)$ & $50.0(28 \sim 66)$ & \\
\hline Age Group & & & 0.986 \\
\hline$\leq 35$ & 10 & 10 & \\
\hline $36 \sim 55$ & 64 & 63 & \\
\hline$>55$ & 24 & 25 & \\
\hline $\mathrm{BSA}, \mathrm{m}^{2}$ & 1.73 & 1.74 & \\
\hline Mean \pm SD & $1.72 \pm 0.12$ & $1.74 \pm 0.11$ & 0.499 \\
\hline Median (range) & $1.71(1.43 \sim 2.01)$ & $1.72(1.45 \sim 2.06)$ & \\
\hline History of hypertension & 14 & 12 & 0.674 \\
\hline Tumor size $(\mathrm{cm})$ & & & 0.907 \\
\hline$\leq 2.0$ & 48 & 51 & \\
\hline $2.1 \sim 5.0$ & 45 & 42 & \\
\hline$>5.0$ & 5 & 5 & \\
\hline Grade & & & 0.827 \\
\hline G1 & 5 & 4 & \\
\hline $\mathrm{G} 2$ & 44 & 48 & \\
\hline G3 & 49 & 46 & \\
\hline ER positive & 47 & 52 & 0.475 \\
\hline PR positive & 39 & 42 & 0.663 \\
\hline p53 positive & 56 & 63 & 0.306 \\
\hline Ki-67 high $(\geq 14 \%)$ & 81 & 76 & 0.371 \\
\hline Lymph nodes & & & 0.867 \\
\hline 0 & 38 & 37 & \\
\hline $1 \sim 3$ & 25 & 28 & \\
\hline $4 \sim 9$ & 13 & 15 & \\
\hline$>9$ & 22 & 18 & \\
\hline AJCC stage & & & 0.839 \\
\hline I & 24 & 23 & \\
\hline II & 38 & 42 & \\
\hline III & 36 & 33 & \\
\hline Chemotherapy Regimen & & & 0.539 \\
\hline Anthracyclines, no taxanes & 33 & 29 & \\
\hline $\begin{array}{l}\text { Anthracyclines plus } \\
\text { taxanes }\end{array}$ & 65 & 69 & 0.059 \\
\hline
\end{tabular}

(Continued) 


\begin{tabular}{lccc}
\hline Variable, $\mathbf{N}(\%)$ & $\begin{array}{c}\text { Concurrent group } \\
(\mathbf{n}=\mathbf{9 8})\end{array}$ & $\begin{array}{c}\text { Sequential group } \\
(\mathbf{n}=\mathbf{9 8})\end{array}$ & $\boldsymbol{P}$-value \\
\hline Concurrent & 37 & 28 & \\
$\quad$ Sequential & 28 & 41 & \\
Cumulative anthracyclines & 361.2 & 359.1 & 0.869 \\
dose $\left(\mathrm{mg} / \mathrm{m}^{2}\right)$ & 43 & 48 & 0.474 \\
Radiotherapy & 24 & 23 & \\
$\quad$ Left-sided tumor & 51 & 55 & 0.566 \\
Endocrine Therapy & & & \\
\hline
\end{tabular}

SD, standard deviation; BSA, body surface area; ER, estrogen receptor; PR, progesterone receptor.

\section{Cardiac safety and other safety}

The cardiac safety events were graded according to the NCI-CTC, version 2.0. Trastuzumab and anthracyclines was well tolerated in both the concurrent group and the sequential group. There was no patient documented congestive heart failure or cardiac death. The primary cardiac safety event in both treatment groups was an asymptomatic decrease in the left ventricular ejection fraction. Nineteen patients $(19.4 \%, 95 \%$ CI $12.4-28.9)$ in the concurrent group and 22 patients $(22.4 \%, 95 \% \mathrm{CI}$ 14.9-32.2) in the sequential group met the criteria for cardiac events (odds ratio $0.831,95 \%$ CI $0.417-1.656, P=$ 0.598 , Table 2). No difference between the two groups was found in the mean LVEF determined at baseline, every 3 months during trastuzumab administration, and at 24 months after the first dose of trastuzumab (Figure 2). In the concurrent group, more than $10 \%$ but less than $20 \%$ reduction in LVEF (NCI-CTC Grade 1) was detected in 17 patients (17.3\%, 95\% CI 10.7-26.6), whereas a reduction higher than $20 \%$ in LVEF or a decline below $50 \%$ (NCICTC Grade 2) was established in two patients $(2.0 \%$, $95 \%$ CI $0.3-7.9$ ). On the other hand, in the sequential group, 21 patients $(21.4 \%, 95 \%$ CI 14.0-31.1) exhibited a Grade 1 LVEF reduction, and 1 patient $(1.0 \%, 95 \%$ CI 0.1-6.4) manifested a Grade 2 reduction. Trastuzumab was temporarily withheld in two participants in the concurrent group and one patient in the sequential group with Grade 2 LVEF reduction. The LVEF values of these three patients recovered in four weeks, and trastuzumab administration was continued. Trastuzumab was permanently discontinued in one patient in the concurrent group after the application of 14 doses because of hearing loss.

\section{Disease-free survival and overall survival}

Fifteen recurrent events were observed in the concurrent group and 16 events in the sequential group (Table 3 and Figure 3). The percentages of patients alive and disease-free at five years were $84.5 \%$ in the concurrent group and $79.4 \%$ in the sequential group (hazard ratio,
$0.956 ; 95 \%$ CI $0.471-1.939 ; P=0.901)$. There was no significant difference between the rates of DFS in the two groups.

There were 14 deaths occurred during follow-up, eight in the concurrent group and six in the sequential group. The Kaplan-Meier estimates of the OS at five years was $91.2 \%$ in the concurrent group and $92.4 \%$ in the sequential group (hazard ratio, $1.443 ; 95 \%$ CI 0.500 $4.167 ; P=0.495$; Figure 3 ). The difference was also nonsignificant.

\section{DISCUSSION}

To our knowledge, this is the first randomised and controlled trial that demonstrated the safety of the concurrent use of trastuzumab and anthracyclines in the adjuvant treatment of early breast cancer. With a median follow-up time of 42 months, $19.4 \%$ of the patients in the concurrent group and $22.4 \%$ in the sequential group met the criteria for cardiac events. There was no patient documented congestive heart failure or cardiac death. No significant difference was found in cardiac safety event rates between the two groups $(P=0.598)$. A slight decline in the mean LVEF values was observed during the administration of trastuzumab; however, they recovered in both groups within one year.

Although the concurrent use of trastuzumab and anthracyclines caused unacceptably high rates of cardiac dysfunction in the metastatic setting [7], this regimen was proven fairly safe in the neoadjuvant setting $[8,9]$. No prospective randomised trial to investigate the cardiac safety of the concurrent regimen of trastuzumab and anthracyclines in the adjuvant treatment of early breast cancer has been reported before. Only one Japanese study by Watanabe and colleagues $[14,15]$ retrospectively reviewed the cardiac tolerability of the concurrent administration of trastuzumab and anthracyclines. In that analysis, 49 patients with HER2-positive breast cancer received paclitaxel (P) followed by epirubicin, fluorouracil, and cyclophosphamide (FEC) and concurrent 
Table 2: Cardiac events during treatment and follow-up

\begin{tabular}{|c|c|c|c|}
\hline & $\begin{array}{l}\text { Concurrent group } \\
(\mathrm{N}=98)\end{array}$ & $\begin{array}{l}\text { Sequential group } \\
(\mathrm{N}=98)\end{array}$ & $P$-value \\
\hline LVEF reduction & $19(19.4)$ & $22(22.4)$ & 0.598 \\
\hline NCI-CTC grade $1^{\dagger}$ & $17(17.3)$ & $21(21.4)$ & \\
\hline NCI-CTC grade 2 & $2(2.0)$ & $1(1.0)$ & \\
\hline NCI-CTC grade $3^{*}$ & 0 & 0 & \\
\hline NCI-CTC grade $4^{*}$ & 0 & 0 & \\
\hline Cardiac death & 0 & 0 & \\
\hline
\end{tabular}

LVEF, left ventricular ejection fraction; NCI-CTC, National Cancer Institute common toxicity criteria.

Data are $\mathrm{n}(\%)$. ${ }^{\dagger}$ Asymptomatic reduction of $10 \%$ or more, but less than $20 \%$ of baseline. ${ }^{*}$ Asymptomatic reduction to lower limit of normal or $20 \%$ or more of baseline. ${ }^{*}$ Congestive heart failure, responsive to treatment. ${ }^{*}$ Refractory or poorly controlled heart failure due to drop in ejection fraction. Lower limit of normal:EF value $=50 \%$.

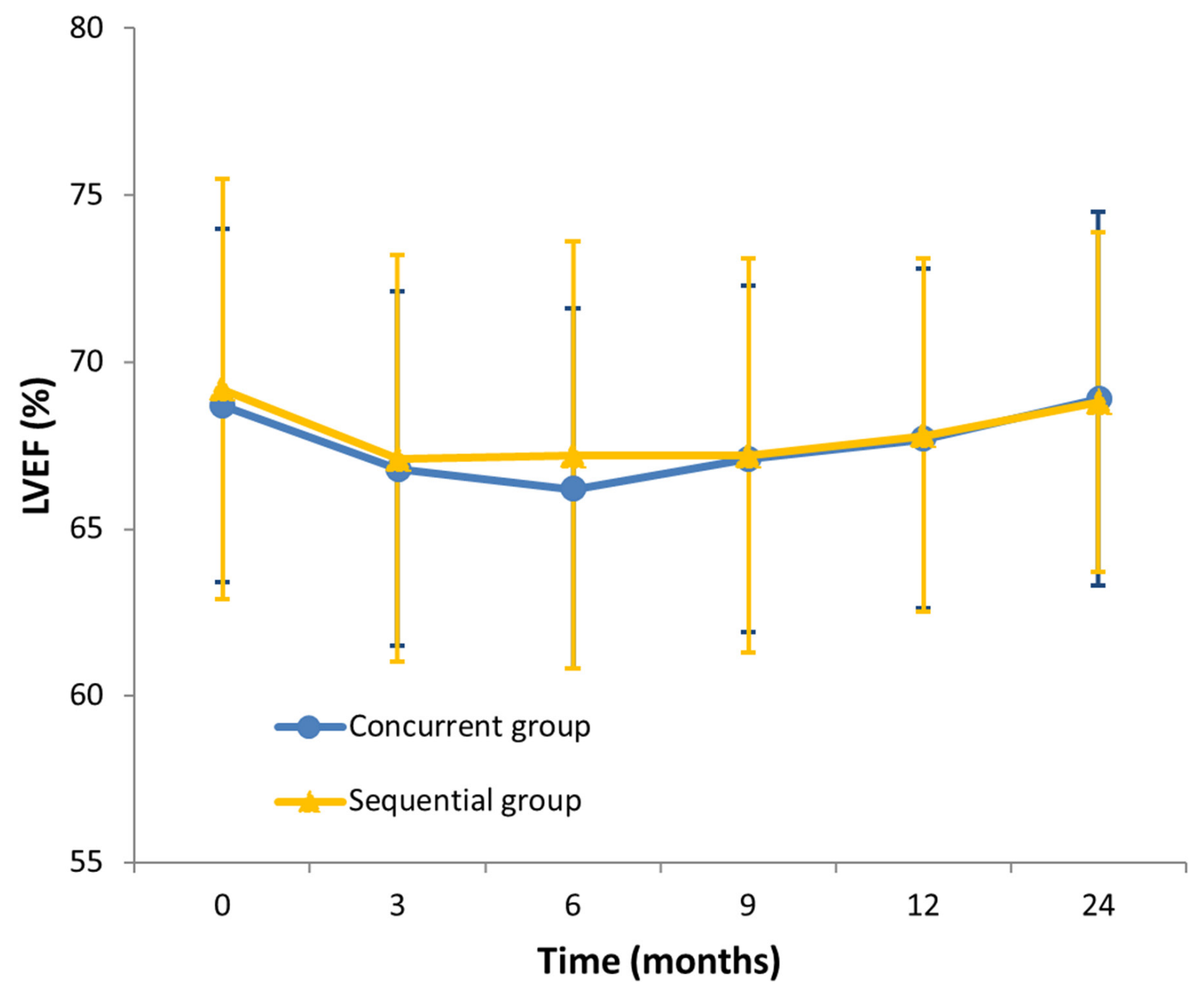

Figure 2: Mean left ventricular ejection fraction (LVEF) measurements during treatment and follow-up. Vertical bars represent standard deviation. 
Table 3: First disease-free survival events by trial arms

\begin{tabular}{|c|c|c|}
\hline & $\begin{array}{l}\text { Concurrent group } \\
(\mathrm{N}=98)\end{array}$ & $\begin{array}{c}\text { Sequential group } \\
(\mathrm{N}=98)\end{array}$ \\
\hline Locoregional recurrence & 4 & 3 \\
\hline Chest wall & 1 & 0 \\
\hline Lymph nodes & 3 & 3 \\
\hline Distant metastasis & 12 & 13 \\
\hline Bone & 4 & 7 \\
\hline Brain & 4 & 1 \\
\hline Liver & 1 & 3 \\
\hline Lung & 3 & 2 \\
\hline Total DFS events & $15^{*}$ & 16 \\
\hline
\end{tabular}

${ }^{*}$ One case presented with both bone and lymph nodes involvement as first manifestation of recurrence.

trastuzumab (Trastuzumab Group). The LVEF values of the patients in the Trastuzumab Group was assessed regularly for more than one year and compared with those of HER2-negative breast cancer patients who received $\mathrm{P}$ followed by FEC (Standard Group). Twelve patients (24.9\%) in the Trastuzumab Group had a LVEF reduction of more than $10 \%$, compared to $22.2 \%$ in the Standard Group $[14,15]$. There was no significant difference in LVEF reduction between the two groups $(P=0.386)[14$, 15]. The cardiac event rate of this retrospective study was similar to that of our trial, which supported our conclusion regarding the cardiac safety of the concurrent administration of trastuzumab and anthracyclines.

Nevertheless, it is unclear whether there is anthracycline-trastuzumab interaction contributing to cardiotoxicity, although some possible mechanisms have been proposed [16, 17]. It is currently considered that anthracyclines cause type I cardiac damage, which is dose-related and associated with identifiable ultrastructural abnormalities, and begins from the initial administration of the drug [18]. While trastuzumab-related cardiotoxicity is classified as Type II, which is highly reversible, non-dosedependent, and unassociated with ultrastructural changes [18]. Since the cardiac toxicity caused by anthracyclines persists during the sequential use of trastuzumab, we propose that the timing of trastuzumab administration is not related to the severity of cardiotoxicity. The high rate of cardiac failure in the metastatic setting might not be caused by the concurrent regimen of trastuzumab and anthracyclines, but by the high cumulative dose of anthracyclines and poor baseline cardiac conditions of the metastatic patients. Therefore, there suggested a high possibility the cardiotoxicity rate would not change even trastuzumab was given sequentially in that trial. The absence of observations of a high rate of cardiotoxicity in all other related trials also provides evidence in support of our interpretation.
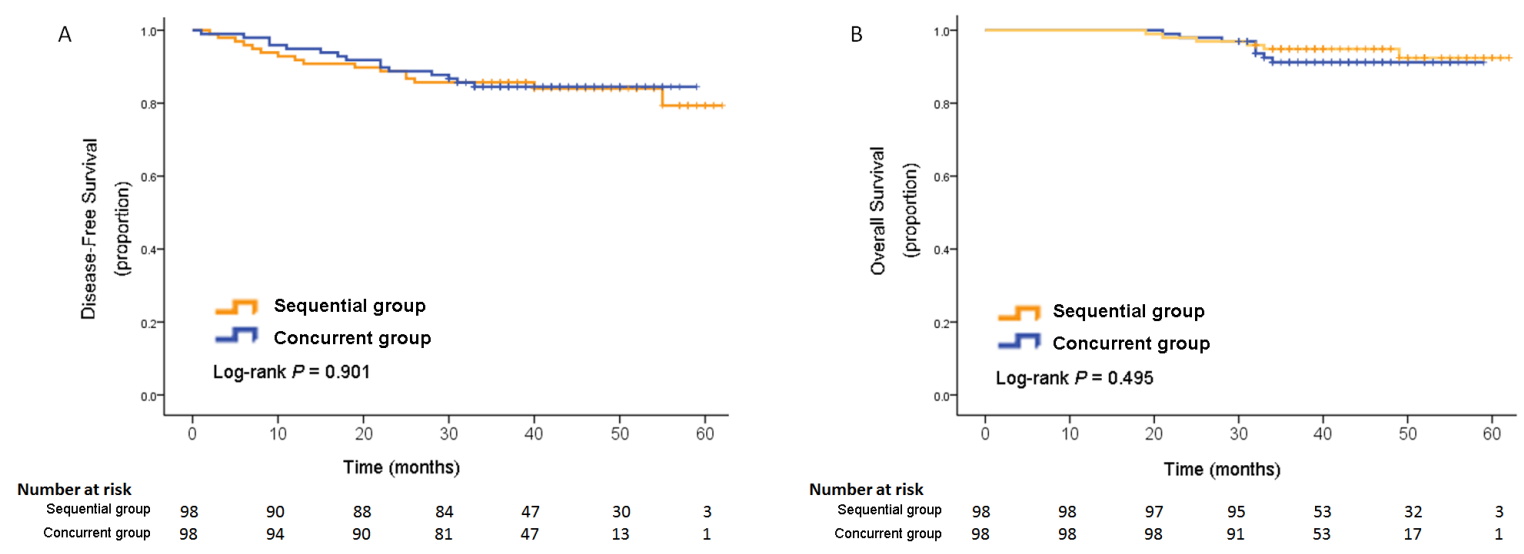

Figure 3: Kaplan-Meier estimates of disease-free survival (A) and overall survival (B). 
Apart from the well-tolerated concurrent administration of trastuzumab and anthracyclines in the adjuvant treatment of breast cancer, there are at least two other reasons to prefer this regimen.

Firstly, the required treatment duration is the shortest of all regimens containing both trastuzumab and anthracyclines, and all phases of this regimen can be completed within 12 months. This will shorten the duration of treatment by 3-6 months, and reduce considerably hospitalization times and costs.

Secondly, the genes encoding topoisomerase II alpha and HER2, the targets for anthracyclines and trastuzumab, respectively, are frequently co-amplified in breast cancers [19]. Pre-clinical studies showed there were additive and/or synergistic therapeutic effects between anthracyclines and trastuzumab [20]. The findings of some trials also suggested that the HER2 status was a predictor of responsiveness to adjuvant anthracyclines administration, and patients with HER2-positive breast tumor might derive preferential benefit from the administration of anthracyclines [46]. The concurrent administration of trastuzumab and anthracyclines in neoadjuvant trials has resulted in high rates of pCR and prolonged survival [8-10].

One limitation of our trial is the relatively small number of patients to show the secondary endpoint of survival benefits. However, the safety issue has priority over the effect, and for more than a decade, cardiac safety has been the obstacle that prevented clinical researchers from investigating the effect of the concurrent administration of trastuzumab and anthracyclines in the adjuvant treatment of breast cancer. The present trial has provided the first evidence of cardiac safety, but larger trials are warranted to confirm the effect.

Concurrent administration of trastuzumab and anthracyclines is a safe adjuvant regimen with a low frequency of cardiac dysfunction, although careful cardiac monitoring is needed. Due to the limitation of the relatively small sample size and short follow-up time, further multicenter clinical trials are required to determine the cardiac safety of the concurrent regimen and whether it improves the survival of patients.

\section{MATERIALS AND METHODS}

\section{Study design}

The present study was a prospective, randomised, and controlled trial in patients with newly diagnosed early breast cancer from Peking Union Medical College Hospital (PUMCH). At the time of registration, the patients with histopathologically confirmed Her2-positive early breast cancer had undergone definitive surgery and planned to receive anthracycline-based adjuvant chemotherapy and trastuzumab treatment. The primary study endpoint was cardiac safety, including symptoms, electrocardiogram, and LVEF changes. Secondary endpoints involved DFS and OS. DFS was defined as the interval from randomization to: local, regional, or distant recurrence; second primary cancer; contralateral breast cancer; or death from any cause, whichever came first. OS was defined as the time from random assignment to death from any cause.

The study was approved by the Institutional Review Board of PUMCH. All patients provided written informed consent. The study was conducted in accordance with the Declaration of Helsinki.

This study was registered at the ClinicalTrials.gov (Identifier: NCT01413828).

\section{Eligibility criteria}

Eligibility requirements included primary operable node-positive or high-risk node-negative invasive breast cancer. All tumors had to be removed by definitive surgery within 60 days of trial registration, and neoadjuvant treatments were not permitted.

All the patients had HER2-positive breast cancer, which was defined as immunohistochemistry (IHC) score of 3+ (Herceptest, Dako, Denmark) or gene amplified by fluorescence in situ hybridization (FISH, Pathvysion HER2 test, Abbott-Vysis, USA) according to the PUMCH laboratory. Participants were excluded if they had locally advanced or metastatic breast cancer, bilateral breast cancer, previous or concurrent any other malignant disease, previous administration of anthracyclines and/or trastuzumab for any disease.

Only adult female patients with adequate hepatic, renal, and bone marrow functions were included as participants in the investigation. An additional requirement for enrollment included having a LVEF value of $55 \%$ or higher measured by echocardiography. Patients were ineligible if they had a history of documented congestive heart failure, angina pectoris, unstable arrhythmias, coronary artery disease with previous myocardial infarction, clinically significant valvular disease, and uncontrolled hypertension.

\section{Randomisation and masking}

Eligible participants were randomly assigned, in a $1: 1$ ratio, to receive adjuvant therapy with trastuzumab administered concurrently with anthracyclines (concurrent group) or sequentially to anthracyclines (sequential group). The chemotherapy regimen and radiation therapy was chosen before randomisation. Randomisation was done by a computer-generated randomization sequence, using a permuted-block randomisation method with a block size of four.

\section{Treatment regimens}

Eligible participants were randomised after definitive surgery to receive concurrent or sequential 
administration of trastuzumab and anthracyclines. The regimens of chemotherapy consisting of at least four cycles of anthracycline-based treatment were chosen at the discretion of their physician based on the National Comprehensive Cancer Network guidelines before randomisation. If anthracyclines were given alone or followed by taxanes, the starting doses of doxorubicin and epirubicin were $60 \mathrm{mg} / \mathrm{m}^{2}$ and $90-100 \mathrm{mg} / \mathrm{m}^{2}$, respectively. Alternatively, if anthracyclines were administered concurrently with taxanes, doses of $50 \mathrm{mg} / \mathrm{m}^{2}$ of doxorubicin and $75 \mathrm{mg} / \mathrm{m}^{2}$ of epirubicin were employed. In all cases, the maximum cumulative allowable doses were $360 \mathrm{mg} / \mathrm{m}^{2}$ of doxorubicin and $600 \mathrm{mg} / \mathrm{m}^{2}$ of epirubicin. The anthracyclines were given every two or three weeks.

Trastuzumab was administered intravenously, beginning with a loading dose of $8 \mathrm{mg} / \mathrm{kg}$ of body weight, and followed by maintenance doses of $6 \mathrm{mg} / \mathrm{kg}$ given every three weeks for one year. If the treatment of trastuzumab was delayed by more than one week, the administration was restarted with the initial dose of $8 \mathrm{mg} /$ $\mathrm{kg}$, followed by the usual maintenance dose $(6 \mathrm{mg} / \mathrm{kg}$ every three weeks).

Radiation therapy was required after completion of chemotherapy to patients who underwent breastconserving surgery or mastectomy with at least four positive nodes. Patients with hormone receptor-positive disease were given adjuvant endocrine therapy after chemotherapy unless contraindicated. Trastuzumab treatment was continued during radiotherapy and/ or endocrine therapy. No cardioprotective drugs (dexrazoxane, angiotensin-converting enzyme inhibitors, beta blockers, et al.) were permitted to use prophylactically.

\section{Cardiac safety}

In addition to monitoring general safety, an intense cardiac monitoring was also scheduled. Cardiac events were graded according to the National Cancer Institute Common Toxicity Criteria, version 2.0 (NCI-CTC, version 2.0). Cardiac monitoring in the concurrent group and the sequential group included history-taking, a 12lead electrocardiogram, and an assessment of LVEF by echocardiography at baseline and at 3, 6, 9, 12, and 24 months after the first dose of trastuzumab. Thereafter, history-taking and physical examination were scheduled every 6 months until the end of the study. Additional scans could be performed at the investigator's discretion. Cardiac safety was monitored by independent cardiologists.

If the absolute value of LVEF declined by $20 \%$ or more from baseline or below $50 \%$, trastuzumab was temporarily discontinued for four weeks during which the LVEF value was reassessed. The administration of trastuzumab was permanently discontinued in case that LVEF values remained below these levels or the participant experienced symptomatic cardiac failure.

\section{Statistical design and analysis}

In the present trial, the primary end point was cardiac safety, defined as the incidence of an asymptomatic LVEF reduction of $10 \%$ absolutely or more from baseline, or below $50 \%$, or symptomatic heart failure. To calculate the sample size, we had to assume the rate of cardiac safety events in each of the two groups, whose values can vary due to the different definitions and populations used in each specific trial. We adopted the rate of cardiac dysfunction of the Breast Cancer International Research Group 006 trial, in which $18.6 \%$ of participants in the sequential group had a LVEF reduction of more than $10 \%$ [3]. According to a trial in the metastatic setting, in which prohibition of the concurrent administration of trastuzumab and anthracyclines in clinical practice was recommended, the rate of heart failure in the concurrent group was increased to $27 \%$ [7]. Thus, we can infer a much higher rate of asymptomatic LVEF reduction of the concurrent administration of trastuzumab and anthracyclines since asymptomatic LVEF reduction is much more common than heart failure. In our investigation, if we were to assume rates of cardiac events in the sequential group and the concurrent group of $19 \%$ and $32 \%$, respectively, 176 participants would have been needed to achieve a twosided statistical significance of $5 \%$ with $80 \%$ power.

Categorical data were compared using the twotailed chi-square test. When the expected counts were low, we employed the Fisher's exact test. Quantitative data were compared by Student's $t$-test. The DFS and OS were estimated using the Kaplan-Meier method. Twosided log-rank test for time-to event endpoints were used. Differences were considered significant at $P<0.05$.

\section{Author contributions}

All authors have reviewed the data interpretation, prepared the report, and approved the final version of the submitted manuscript. QS and SS participated in the study design. YX was involved in statistical planning and data analysis.

\section{ACKNOWLEDGMENTS}

We thank all the patients who have participated in our trial, and the medical teams for their cooperation.

\section{CONFLICTS OF INTEREST}

The authors declare that they have no conflicts of interest.

\section{FUNDING}

This work was supported by the Beijing Natural Science Foundation (7172168), Beijing Municipal 
Science and Technology Key Development Program (D090507043409009) and the Twelfth Five-year Key Programs for Science and Technology Development of China (2014BAI08B03).

\section{REFERENCES}

1. Gianni L, Dafni U, Gelber RD, Azambuja E, Muehlbauer S, Goldhirsch A, Untch M, Smith I, Baselga J, Jackisch C, Cameron D, Mano M, Pedrini JL, et al. Treatment with trastuzumab for 1 year after adjuvant chemotherapy in patients with HER2-positive early breast cancer: a 4-year follow-up of a randomised controlled trial. Lancet Oncol. 2011; 12:236-44. https://doi.org/10.1016/ S1470-2045(11)70033-X.

2. Perez EA, Romond EH, Suman VJ, Jeong JH, Davidson NE, Geyer CE Jr, Martino S, Mamounas EP, Kaufman PA, Wolmark N. Four-year follow-up of trastuzumab plus adjuvant chemotherapy for operable human epidermal growth factor receptor 2-positive breast cancer: joint analysis of data from NCCTG N9831 and NSABP B-31. J Clin Oncol. 2011; 29:3366-73. https://doi.org/10.1200/ JCO.2011.35.0868

3. Slamon D, Eiermann W, Robert N, Pienkowski T, Martin M, Press M, Mackey J, Glaspy J, Chan A, Pawlicki M, Pinter T, Valero V, Liu MC, et al. Adjuvant trastuzumab in HER2positive breast cancer. N Engl J Med. 2011; 365:1273-83. https://doi.org/10.1056/NEJMoa0910383.

4. Rayson D, Richel D, Chia S, Jackisch C, van der Vegt S, Suter T. Anthracycline-trastuzumab regimens for HER2/ neu-overexpressing breast cancer: current experience and future strategies. Ann Oncol. 2008; 19:1530-9. https://doi. org/10.1093/annonc/mdn292.

5. Gennari A, Sormani MP, Pronzato P, Puntoni M, Colozza M, Pfeffer U, Bruzzi P. HER2 status and efficacy of adjuvant anthracyclines in early breast cancer: a pooled analysis of randomized trials. J Natl Cancer Inst. 2008; 100:14-20. https://doi.org/djm252.

6. Bartlett JM, Munro AF, Dunn JA, McConkey C, Jordan S, Twelves CJ, Cameron DA, Thomas J, Campbell FM, Rea DW, Provenzano E, Caldas C, Pharoah P, et al. Predictive markers of anthracycline benefit: a prospectively planned analysis of the UK National Epirubicin Adjuvant Trial (NEAT/BR9601). Lancet Oncol. 2010; 11:266-74. https:// doi.org/S1470-2045(10)70006-1.

7. Slamon DJ, Leyland-Jones B, Shak S, Fuchs H, Paton V, Bajamonde A, Fleming T, Eiermann W, Wolter J, Pegram M, Baselga J, Norton L. Use of chemotherapy plus a monoclonal antibody against HER2 for metastatic breast cancer that overexpresses HER2. N Engl J Med. 2001; 344:783-92. https://doi.org/10.1056/ NEJM200103153441101.

8. Gianni L, Eiermann W, Semiglazov V, Manikhas A, Lluch A, Tjulandin S, Zambetti M, Vazquez F, Byakhow
M, Lichinitser M, Climent MA, Ciruelos E, Ojeda B, et al. Neoadjuvant chemotherapy with trastuzumab followed by adjuvant trastuzumab versus neoadjuvant chemotherapy alone, in patients with HER2-positive locally advanced breast cancer (the NOAH trial): a randomised controlled superiority trial with a parallel HER2-negative cohort. Lancet. 2010; 375:377-84. https://doi.org/ S0140-6736(09)61964-4.

9. Buzdar AU, Valero V, Ibrahim NK, Francis D, Broglio KR, Theriault RL, Pusztai L, Green MC, Singletary SE, Hunt KK, Sahin AA, Esteva F, Symmans WF, et al. Neoadjuvant therapy with paclitaxel followed by 5-fluorouracil, epirubicin, and cyclophosphamide chemotherapy and concurrent trastuzumab in human epidermal growth factor receptor 2-positive operable breast cancer: an update of the initial randomized study population and data of additional patients treated with the same regimen. Clin Cancer Res. 2007; 13:228-33. https:// doi.org/13/1/228.

10. Gianni L, Eiermann W, Semiglazov V, Lluch A, Tjulandin S, Zambetti M, Moliterni A, Vazquez F, Byakhov MJ, Lichinitser M, Climent MA, Ciruelos E, Ojeda B, et al. Neoadjuvant and adjuvant trastuzumab in patients with HER2-positive locally advanced breast cancer (NOAH): follow-up of a randomised controlled superiority trial with a parallel HER2-negative cohort. Lancet Oncol. 2014; 15:640-7. https://doi.org/10.1016/S1470-2045(14)70080-4.

11. Buzdar AU, Suman VJ, Meric-Bernstam F, Leitch AM, Ellis MJ, Boughey JC, Unzeitig G, Royce M, McCall LM, Ewer MS, Hunt KK; American College of Surgeons Oncology Group investigators. Fluorouracil, epirubicin, and cyclophosphamide (FEC-75) followed by paclitaxel plus trastuzumab versus paclitaxel plus trastuzumab followed by FEC-75 plus trastuzumab as neoadjuvant treatment for patients with HER2-positive breast cancer (Z1041): a randomised, controlled, phase 3 trial. Lancet Oncol. 2013; 14:1317-25. https://doi.org/10.1016/S1470-2045(13)70502-3.

12. Perez EA, Suman VJ, Davidson NE, Gralow JR, Kaufman PA, Visscher DW, Chen B, Ingle JN, Dakhil SR, Zujewski J, Moreno-Aspitia A, Pisansky TM, Jenkins RB. Sequential versus concurrent trastuzumab in adjuvant chemotherapy for breast cancer. J Clin Oncol. 2011; 29:4491-7. https:// doi.org/JCO.2011.36.7045.

13. Denduluri N, Somerfield MR, Eisen A, Holloway JN, Hurria A, King TA, Lyman GH, Partridge AH, Telli ML, Trudeau ME, Wolff AC. Selection of optimal adjuvant chemotherapy regimens for human epidermal growth factor receptor 2 (HER2) -negative and adjuvant targeted therapy for HER2-positive breast cancers: An American Society of Clinical Oncology Guideline Adaptation of the Cancer Care Ontario Clinical Practice Guideline. J Clin Oncol. 2016; 34:2416-27. https://doi.org/10.1200/JCO.2016.67.0182.

14. Watanabe N, Otsuka S, Sasaki Y, Shimojima R, Wani Y, Uchino K. Cardiac tolerability of concurrent administration 
of trastuzumab and anthracycline-based regimen as adjuvant chemotherapy for breast cancer. Breast Care (Basel). 2014; 9:46-51. https://doi.org/10.1159/000358754.

15. Watanabe N, Otsuka S, Sasaki Y, Yuasa T, Shimada K. Retrospective analysis of cardiac tolerability of concurrent administration of trastuzumab and anthracycline-based regimen for breast cancer, to address one-year-term issues in LVEF. Breast Dis. 2015; 35:253-61. https://doi. org/10.3233/BD-150418.

16. Romond EH, Jeong JH, Rastogi P, Swain SM, Geyer CE Jr, Ewer MS, Rathi V, Fehrenbacher L, Brufsky A, Azar CA, Flynn PJ, Zapas JL, Polikoff J, et al. Sevenyear follow-up assessment of cardiac function in NSABP $\mathrm{B}-31$, a randomized trial comparing doxorubicin and cyclophosphamide followed by paclitaxel (ACP) with ACP plus trastuzumab as adjuvant therapy for patients with nodepositive, human epidermal growth factor receptor 2-positive breast cancer. J Clin Oncol. 2012; 30:3792-9. https://doi. org/10.1200/JCO.2011.40.0010.
17. Ewer MS. The anthracycline-trastuzumab interaction: up-regulated binding may provide vital mechanistic insight. Eur J Cancer. 2007; 43:2024-5. https://doi.org/10.1016/j. ejca.2007.07.008.

18. Ewer MS, Lippman SM. Type II chemotherapy-related cardiac dysfunction: time to recognize a new entity. J Clin Oncol. 2005; 23:2900-2. https://doi.org/23/13/2900.

19. Jarvinen TA, Tanner M, Rantanen V, Barlund M, Borg A, Grenman S, Isola J. Amplification and deletion of topoisomerase IIalpha associate with ErbB-2 amplification and affect sensitivity to topoisomerase II inhibitor doxorubicin in breast cancer. Am J Pathol. 2000; 156:839-47.

20. Pegram M, Hsu S, Lewis G, Pietras R, Beryt M, Sliwkowski M, Coombs D, Baly D, Kabbinavar F, Slamon D. Inhibitory effects of combinations of HER-2/neu antibody and chemotherapeutic agents used for treatment of human breast cancers. Oncogene. 1999; 18:2241-51. https://doi. org/10.1038/sj.onc.1202526. 